\title{
Postnatal steroid management in preterm infants with evolving bronchopulmonary dysplasia
}

\author{
Zeyar T. Htun ${ }^{1} \cdot$ Elizabeth V. Schulz $\mathbb{1}^{2} \cdot$ Riddhi K. Desai ${ }^{1} \cdot$ Jaime L. Marasch $\mathbb{1}^{1,3} \cdot$ Christopher C. McPherson $\mathbb{(}^{4,5}$ • \\ Lucy D. Mastrandrea ${ }^{6}$ Alan H. Jobe $\mathbb{1}^{7} \cdot$ Rita M. Ryan ${ }^{1}{ }^{1}$
}

Received: 12 January 2021 / Revised: 6 April 2021 / Accepted: 28 April 2021 / Published online: 19 May 2021

This is a U.S. government work and not under copyright protection in the U.S.; foreign copyright protection may apply 2021

\begin{abstract}
Bronchopulmonary dysplasia (BPD) is a chronic lung disease commonly affecting extremely preterm infants. Although mechanical ventilation and oxygen requirements in premature infants are identified as inciting mechanisms for inflammation and the development of BPD over time, data now support an array of perinatal events that may stimulate the inflammatory cascade prior to delivery. Corticosteroids, such as dexamethasone and hydrocortisone, have proven beneficial for the prevention and management of BPD postnatally due to their anti-inflammatory characteristics. This review aims to examine the pharmacologic properties of several corticosteroids, appraise the existing evidence for postnatal corticosteroid use in preterm infants, and assess steroid management strategies to ameliorate BPD. Finally, we aim to provide guidance based on clinical experience for managing adrenal suppression resulting from prolonged steroid exposure since this is an area less well-studied.
\end{abstract}

\section{Introduction}

Bronchopulmonary dysplasia (BPD) is one of the most common morbidities in extremely preterm infants and is strongly associated with poor neurocognitive outcomes [1]. Persistent inflammation, leading to abnormal lung

Elizabeth V. Schulz

elizabeth.schulz@usuhs.edu

1 Division of Neonatology, Department of Pediatrics, UH Rainbow Babies and Children's Hospital, Case Western Reserve University School of Medicine, Cleveland, OH, USA

2 Department of Pediatrics, Uniformed Services University of Health Sciences, Bethesda, MD, USA

3 Department of Pharmacy, Rainbow Babies and Children's Hospital, Cleveland, OH, USA

4 Department of Pharmacy, St. Louis Children's Hospital, St. Louis, MO, USA

5 Department of Pediatrics, Washington University School of Medicine, St. Louis, MO, USA

6 Department of Pediatrics, Jacobs School of Medicine and Biomedical Sciences, University at Buffalo, Buffalo, NY, USA

7 Cincinnati Children's Hospital Medical Center, Division of Neonatology, Pulmonary Biology, University of Cincinnati, Cincinnati, OH, USA development characterized by alveolar simplification and fibrosis, plays a crucial role in BPD development [2]. The inflammatory origins of BPD are multidimensional. Inflammatory cell recruitment can begin in utero, in the setting of clinical or histologic chorioamnionitis. Postnatally, several factors may compound the inflammatory process, such as sepsis, mechanical ventilation, oxygen exposure, and/or intercurrent acute illnesses such as necrotizing enterocolitis [1-3]. These inflammatory insults can lead to recruitment of pro-inflammatory mediators and inflammatory cells (e.g., polymorphonuclear leukocytes, macrophages) within the premature lung tissue. The proinflammatory setting contributes to the various pathologic signs of epithelial necrosis, fibrosis, inappropriate alveolar septation and simplification, and dysregulated microvascular growth seen in evolving BPD [2]. The clinical use of corticosteroids aims to decrease inflammation in the lungs by reducing lymphocyte count, promoting antiinflammatory cytokines, and suppressing pro-inflammatory cytokines by genomic modification [4].

Due to their anti-inflammatory properties, corticosteroids, such as dexamethasone and hydrocortisone, are used to prevent and manage BPD. We will review the pharmacology of corticosteroids ("Steroid pharmacology") and the existing evidence for postnatal corticosteroids use in preterm infants ("Postnatal steroid management in preterm infants"). We 
Fig. 1 Mechanism of corticosteroid effects. After a glucocorticoid crosses the cell membrane it forms a

glucocorticoid-glucocorticoid receptor (GR) complex. This complex is then able to enter the cell nucleus and exert genomic effects that either increase or decrease gene transcription. This complex may also have nongenomic effects by increasing synthesis of annexin $\mathrm{A} 1$ or interacting with cell membrane.

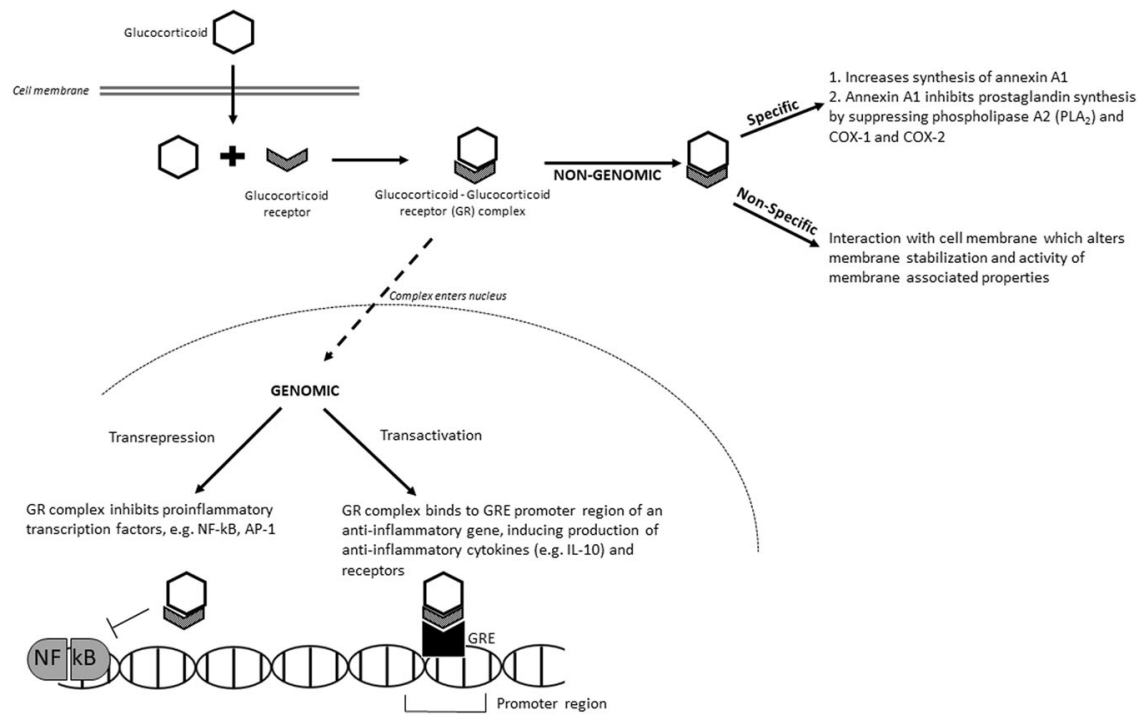

will also recommend steroid management strategies based on the available evidence, using caution for when the evidence is less robust. The evidence available regarding the routine use of inhaled corticosteroids (ICS) for BPD treatment suggests it is not recommended currently; therefore, ICS will have limited discussion in this review.

Finally, a major challenge in using steroids for neonatal lung disease is the concern for adrenal suppression and risk of adrenal insufficiency if glucocorticoids are stopped too rapidly. We will address this problem and possible management strategies for the clinician ("Management of adrenal suppression"). Since there are limited data available providing such guidance, we provide our shared clinical experience in managing adrenal suppression as well as counseling families, follow-up with the caregiver, and monitoring, if needed, once the patient is discharged from the hospital.

\section{Steroid pharmacology}

\section{Mechanism of action and drug properties}

Corticosteroids exert their anti-inflammatory and immunosuppressive effects through genomic mechanisms [5]. They diffuse across cell membranes and bind to receptors, inducing a conformational change of the receptor and formation of the receptor-corticosteroid complex [2, 4, 6]. The receptor-corticosteroid complex enters the cell nucleus. For glucocorticoids, the complex binds to DNA at a glucocorticoid response element (GRE) motif resulting in transactivation (upregulation of anti-inflammatory mediator expression) and transrepression (inhibition of proinflammatory mediator expression). An example of transpression is illustrated by a GRE blocking expression of the nuclear factor $\mathrm{\kappa B}$ or activator protein 1 , both of which are transcription factors responsible for promoting proinflammatory gene expressions (Fig. 1) [5]. Other effects of corticosteroids include inhibition of phospholipase A2 (which is also responsible for the production of proinflammatory mediators) and inhibition of genes responsible for the expression of cyclooxygenase-2 [4].

\section{Glucocorticoid pharmacology}

Glucocorticoids have rapid absorption and generally good oral bioavailability, ranging from 60 to $100 \%$. Endogenously, $\geq 90 \%$ of serum cortisol becomes protein-bound (reversible) with either albumin or transcortin (also known as corticosteroid binding globulin (CBG) or Serpin A6) $[7,8]$. A similar mechanism occurs with synthetic corticosteroids, although the percentage bound to protein varies for each drug [5]. The remaining unbound portion is the biologically active component that traverses the cell membrane and mediates the steroid's molecular effects. At higher steroid concentrations, CBG may become saturated, leading to an increase in unbound steroid available to access the cell [9]. Of note, CBG levels are lower in premature and sick infants, increasing the amount of unbound steroid $[10,11]$. A cortisol level measures the total cortisol level (bound and free). Therefore, cortisol measurements in these infants may overestimate the need for a replacement since it may not accurately reflect the free cortisol level. Glucocorticoids undergo hepatic metabolism through phase 1 oxidation and reduction reactions followed by phase II conjugation. The phase II reactions involved in corticosteroid metabolism include sulfation or glucuronidation. The inactive metabolites are excreted renally (1-20\% excreted as unchanged drug) $[5,9]$. Since maturation of these organ systems are impacted in the premature neonate, further research is needed regarding the pharmacokinetics of 
various systemic glucocorticoids administered to preterm infants for BPD prevention/treatment.

\section{Comparison of corticosteroid agents}

Natural steroids possess both glucocorticoid and mineralocorticoid activity to some degree [12]. Cortisol is the endogenous glucocorticoid produced by the adrenal gland via cholesterol metabolism. Aldosterone is the primary mineralocorticoid produced by the zona glomerulosa of the adrenal gland, with secretion regulated by the reninangiotensin system [4]. The mineralocorticoid effect occurs primarily in the renal epithelial cells and is mediated by intracellular mineralocorticoid receptors which regulate transcription of sodium channels. This pathway enhances sodium absorption and potassium excretion in the nephrons with subsequent passive water reabsorption leading to increased intravascular volume and blood pressure [13].

Synthetically derived corticosteroid analogs have intrinsic properties to mimic and increase glucocorticoid or mineralocorticoid activity and potency. Synthetic structural modifications (Fig. 2) improve glucocorticoid receptor specificity and duration of receptor binding [4]. The basic structure of cortisol includes four basic rings (A-D) and several features important for both glucocorticoid and mineralocorticoid activity. The non-planar orientation of the various molecular groups attached account for the steroid's biological activity [5]. Structural components that are critical for both glucocorticoid and mineralocorticoid activity include the 3-ketone group and the 4,5 double bond on ring A. The presence of the 17-hydroxyl group contributes to both the glucocorticoid function and potency. The 11hydroxyl group is also needed for glucocorticoid activity.

The primary corticosteroids utilized for the antiinflammatory effects in infants with BPD include hydrocortisone, dexamethasone, prednisolone, and methylprednisolone. Although other corticosteroids, such as betamethasone and fludrocortisone, have been considered, there are limited and variable data available to support either drug's place in evolving BPD and these will not be reviewed [14, 15]. The glucocorticoid and mineralocorticoid activity of these corticosteroids are described relative to hydrocortisone, the synthetic analog of cortisol (Table 1). Hydrocortisone primarily possesses glucocorticoid activity, although it exhibits mineralocorticoid activity at supraphysiologic doses. The mineralocorticoid activity is eliminated at physiologic doses $\left(8-10 \mathrm{mg} / \mathrm{m}^{2} /\right.$ day $)$ due to the renal conversion of cortisol to cortisone by $11 \beta$ hydroxysteroid dehydrogenase type $2[5,16]$. The higher doses of hydrocortisone are necessary to alter the dysregulated immune response that contributes to BPD. While supraphysiologic doses of hydrocortisone could have mineralocorticoid effect, the doses used in treating these

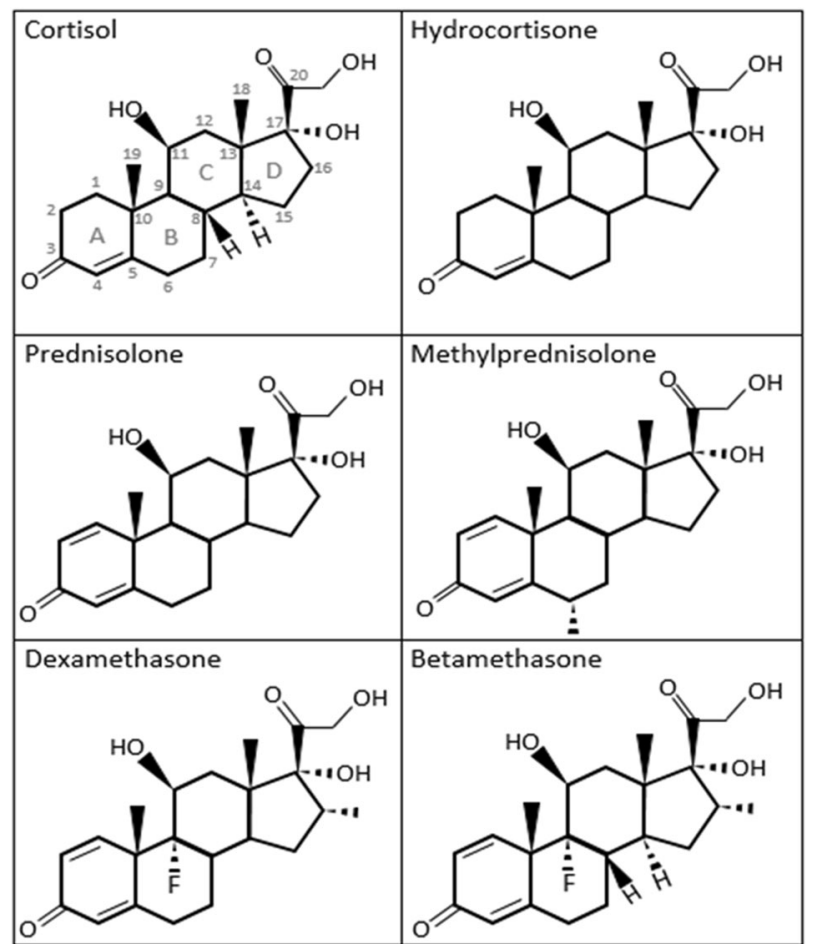

Fig. 2 Molecular structure of endogenous cortisol and other synthetic structural derivatives. The basic structure of cortisol, the body's endogenous corticosteroid, includes four basic rings (A-D) and several features important for both glucocorticoid and mineralocorticoid activity. The non-planar orientation of the various methyl (CH3) and hydroxyl $(\mathrm{OH})$ groups account for the steroid's biological activity [5]. Structural components that are critical for both glucocorticoid and mineralocorticoid activity include the 3-ketone group and the 4,5 double bond on ring A. The presence of the 17-hydroxyl group contributes to both the glucocorticoid function and potency. The 11-hydroxyl group is needed for glucocorticoid, but not mineralocorticoid activity.

infants do not reach the pharmacologic threshold to induce significant sodium retention or hypertension. Pharmacologic doses for BPD prevention or treatment can range from slightly above physiologic doses to greater than recommended stress dosing, and therefore may or may not induce a mineralocorticoid effect (such as hypertension) depending on the dose and duration [14, 17]. Due to its rapid oral absorption and short-acting duration, hydrocortisone is appropriate for physiologic cortisol replacement in the setting of hypothalamic-pituitary-adrenal (HPA) axis suppression that occurs with supraphysiologic doses of glucocorticoids (Table 1) $[4,16]$. This will be discussed further in "Management of adrenal suppression".

Dexamethasone is a long-acting glucocorticoid agonist that is about 25 times more potent than hydrocortisone. The potency of dexamethasone is attributed to its relative stability due to the halogenated structure (carbon 9) and the additional double bond found between carbons 1 and 2 (Fig. 2). Dexamethasone possesses affinity solely for 
Table 1 Comparison of systemic corticosteroids $[4,7,14,16]$.

\begin{tabular}{|c|c|c|c|c|}
\hline Drug & $\begin{array}{l}\text { Approximate } \\
\text { equivalent dose } \\
(\mathrm{mg})^{\mathrm{a}}\end{array}$ & $\begin{array}{l}\text { Glucocorticoid } \\
\text { potency }\end{array}$ & $\begin{array}{l}\text { Mineralocorticoid } \\
\text { potency }\end{array}$ & Duration of action (h) \\
\hline Hydrocortisone & 20 & 1 & 1 & $\begin{array}{l}\text { Short acting } \\
8-12\end{array}$ \\
\hline $\begin{array}{l}\text { Prednisone } \\
\text { Prednisolone }\end{array}$ & 5 & 4 & 0.8 & $\begin{array}{l}\text { Intermediate acting } \\
12-36\end{array}$ \\
\hline Methylprednisolone & 4 & 5 & 0.5 & $\begin{array}{l}\text { Intermediate acting } \\
12-36\end{array}$ \\
\hline Dexamethasone & 0.75 & 25 & 0 & $\begin{array}{l}\text { Long acting } \\
>36\end{array}$ \\
\hline Betamethasone & 0.75 & 25 & 0 & $\begin{array}{l}\text { Long acting } \\
>36\end{array}$ \\
\hline Fludrocortisone & $-{ }^{b}$ & 10 & 125 & $\begin{array}{l}\text { Intermediate acting } \\
12-36\end{array}$ \\
\hline
\end{tabular}

${ }^{a}$ Dose equivalents are reported for oral/IV administration; alternate routes may vary and are not presented here.

${ }^{\mathrm{b}}$ Fludrocortisone is used for mineralocorticoid activity only. albumin (no affinity for transcortin) with $\sim 75 \%$ plasma binding capacity [5]. Unlike other glucocorticoids (e.g., prednisolone), dexamethasone's bioavailability is not subject to processing by $11 \beta$-HSD (type 1 or 2 ). In fact, dexamethasone does not require activation to exert effects on glucocorticoid receptors, and does not bind to mineralocorticoid receptors [18].

Prednisolone and methylprednisolone are synthetic glucocorticoids with similar potency and are used interchangeably in practice. Prednisone is a prodrug that is converted to its active form, prednisolone, by $11 \beta$-HSD1 in the liver [2, 4]. Methylprednisolone, a corticosteroid containing glucocorticoid potency similar to prednisolone, is sometimes used in place of prednisolone when an intravenous formulation is desired. Historically, these corticosteroids have been safely used for other pulmonary diseases, primarily within the pediatric asthma population [19-21].

\section{Postnatal steroid management in preterm infants}

Clinical trials examining the use of postnatal corticosteroids to prevent BPD in preterm infants focus on two time periods (defined by Cochrane reviews): "early" use (0-7 days of age) and "late" use (>7 days of age) [22, 23].

\section{Early use of postnatal corticosteroids (0-7 days of age) to prevent BPD}

Early use of postnatal corticosteroids (birth to 7 days of age) targets relative cortisol insufficiency and mediates inflammatory injury in the perinatal period (such as chorioamnionitis, surfactant insufficiency, and early invasive mechanical ventilation) $[24,25]$. Dexamethasone has been studied most extensively in randomized controlled trials (RCT). Early dexamethasone (within 7 days after birth) decreases the incidence of death or BPD at 36 weeks' postmenstrual age (PMA) (relative risk (RR) 0.87, 95\% confidence interval (95\% CI) 0.80-0.94), and decreases BPD, defined as oxygen supplementation at 36 weeks' PMA (RR 0.71, 95\% CI 0.62-0.81) when compared to placebo [22]. However, these pulmonary benefits are outweighed by the associated short-term adverse effect of gastrointestinal perforation, specifically in conjunction with indomethacin, and long-term neurodevelopmental adverse effects [22]. Specifically, early dexamethasone increases risk for neuromotor impairment and cerebral palsy (CP), even considering treatment courses as short as 3 days $[26,27]$. In this setting, early use ( $<7$ days of age) of dexamethasone cannot be recommended as the short- and long-term adverse effects are concerning and outweigh the benefit of reduction in BPD.

In contrast, emerging data may support the role of early hydrocortisone use for the prevention of BPD. In 2016, the PREMILOC trial $(N=523)$ documented improved survival without BPD at 36 weeks' PMA in premature neonates treated with 10 days of low-dose hydrocortisone starting at $<24 \mathrm{~h}$ of age compared to placebo (60\% vs. $51 \%$, OR 1.48 , 95\% CI [1.02-2.16], $p=0.04$ ) (Table 2) [28]. The PREMILOC trial avoided the potential risk of increased gastrointestinal perforation by prohibiting prophylactic nonsteroidal anti-inflammatory drugs (NSAIDs) in the first $24 \mathrm{~h}$ of life. In contrast to dexamethasone, the PREMILOC trial showed that early low-dose hydrocortisone produces no increased risk for long-term neurodevelopmental impairment (NDI) or CP [29]. In fact, for the subgroup of infants born at 24-25 weeks' gestational age (GA), a 


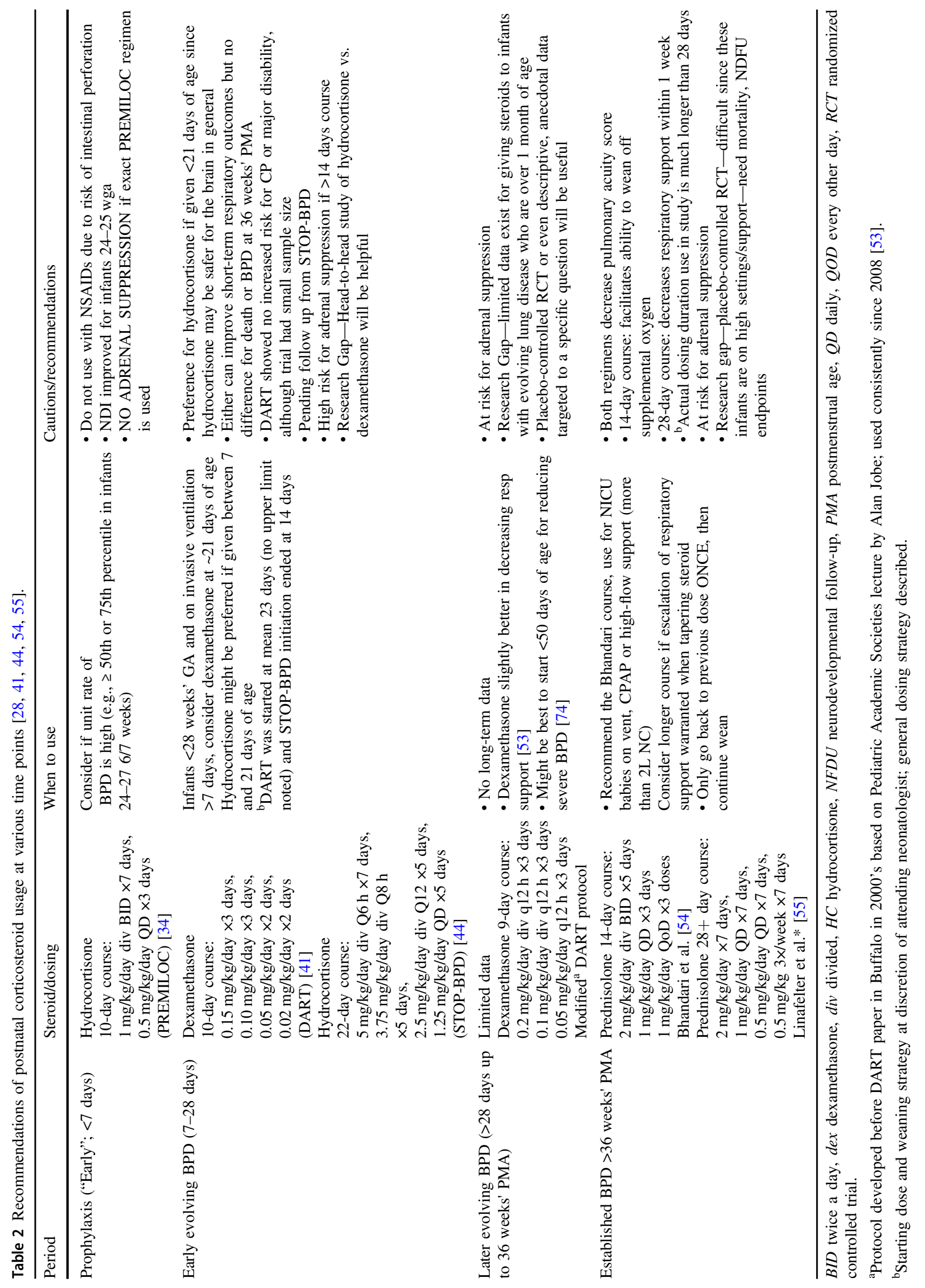


significant improvement in global neurological assessment was observed in post hoc analyses of the hydrocortisone group compared with the placebo group (moderate-tosevere NDI $2 \%$ vs. $18 \%, p=0.02$ ) [30]. Overall, improvements in survival without BPD at 36 weeks' PMA without increasing the risk of NDI support hydrocortisone's role as the early corticosteroid of choice for infants with an unacceptably high risk for pulmonary morbidity.

Administration of ICS may decrease pulmonary inflammation while minimizing systemic exposure. The NEUROSIS trial $(N=863)$ was a multinational, randomized trial that administered high-dose inhaled budesonide or placebo within $24 \mathrm{~h}$ after birth to infants ( $<28$ weeks' GA) until they were off supplemental oxygen or reached 32 weeks' PMA. The trial showed a reduction of BPD for the inhaled budesonide group compared to placebo $(27.8 \%$ vs. $38.0 \%$, $p=0.004$ ) [31]. However, this benefit was offset by a nonsignificant increase in mortality with budesonide compared with placebo $(16.9 \%$ vs. $13.6 \%$, RR 1.24, 95\% CI [0.91-1.69], $p=0.17$ ). No difference was noted in combined mortality or BPD (40\% in inhaled budesonide group vs. $46.3 \%$ in the placebo group, $p=0.05$ ). Although the rate of neurodevelopmental disability did not differ between the groups, the difference in mortality at 2 years of age follow-up was higher for the treatment group (19.9\% vs. $14.5 \%$, RR 1.37, 95\% CI [1.01-1.86], $p=0.04$ ) [32]. Based on these findings, early administration of ICS cannot be recommended at this time.

One of the limitations of ICS in preterm infants is poor pulmonary deposition due to intrinsically low lung volumes and inspiratory pressures $[33,34]$. To overcome this limitation, corticosteroids can be instilled into the lungs using surfactant as a vehicle. Two single-center trials evaluated this approach $[35,36]$. In the larger randomized trial $(N=265)$, very low birth weight (VLBW) neonates who received intratracheal surfactant/budesonide compared with surfactant alone had a significantly lower incidence of BPD or death (55 of 131 [42.0\%] vs. 89 of 134 [66\%], risk ratio $0.58,95 \%$ CI [0.44-0.77], $p<0.001$; number needed to treat $4.1,95 \% \mathrm{CI}$ [2.8-7.8]) [36]. There were no differences in growth parameters or NDI between the intervention and control group at 2 to 3 years of age [36]. This promising single-center data may be bolstered by the ongoing National Institute of Child Health and Human Development (NICHD) funded trial "The Budesonide in Babies (BiB) Trial" [37]. Until further data are produced and a combined corticosteroid/surfactant preparation is commercially available, widespread adoption of this approach is discouraged at this time.

\section{Late use of postnatal corticosteroids ( $>7$ days of age)}

Late ( $>7$ days of age) dexamethasone consistently produces significant reductions in BPD and the combined outcome of death or BPD, along with lower extubation failure rates, need for rescue corticosteroids, and for home oxygen therapy [23]. However, an increased risk of $\mathrm{CP}$ has been associated with late high-dose dexamethasone therapy, although aggregate data analysis has shown no increase in NDI [38-40]. The negative findings were primarily due to the traditional higher and longer dosing courses (21-42-day tapering courses) of dexamethasone. Recent data demonstrate that delaying exposure to corticosteroids until after 7 days of age while utilizing shorter courses and lower doses may favorably shift the risk:benefit ratio [23, 38]. Because the "late" period is expansive, we have identified three periods for discussion: early evolving BPD (7-28 days), late evolving BPD (28 days to 36 weeks' PMA), and established BPD ( $\geq 36$ weeks' PMA).

\section{Late use of postnatal corticosteroids ( $>7$ days of age): early evolving BPD (<28 days)}

To address both the efficacy and safety of late low-dose, short-course dexamethasone, Dexamethasone: A Randomized Trial (DART) studied a cumulative dose of $0.89 \mathrm{mg} /$ $\mathrm{kg}$ over 10 days in extremely low birth weight or very preterm infants older than a week of age (Table 2) [41]. This pivotal trial was hampered by slow recruitment, owing to the widespread backlash against dexamethasone therapy among clinicians and parents concerned about potential neurodevelopmental harm [42]. The median age at which dexamethasone was initiated in this trial was 23 days, but the trial was halted well before achieving the desired sample size. Despite this limitation, the trial revealed that late lowdose dexamethasone compared to placebo was able to facilitate extubation (day 3: $34 \%$ vs. $3 \%, p<0.01$; day 7 : $51 \%$ vs. $12 \%, p<0.01$; day $10: 60 \%$ vs. $12 \%, p<0.01$ ), but did not improve survival or oxygen dependence at 36 weeks' PMA. DART follow-up showed no differences in the rate of major disability, $\mathrm{CP}$, or combined death or $\mathrm{CP}$ at 2 years of age. However, the lack of statistical power renders these findings inconclusive [43].

The STOP-BPD trial $(N=372)$ provides evidence for the emerging utilization of late hydrocortisone therapy for BPD prevention [44]. Preterm infants between 7 and 14 days of age who were ventilator dependent and at high risk for developing BPD were randomized to either high-dose hydrocortisone or placebo (Table 2) [44]. There was no difference in the primary composite outcome of death or BPD at 36 weeks' PMA (71\% vs. $74 \%$, respectively; $p=$ $0.54)$; however, there was a significant reduction in the mortality component of the primary endpoint (15.5\% vs. $23.7 \%, p=0.048)$. Similar to dexamethasone in DART, hydrocortisone compared to placebo facilitated extubation (day 3: $16 \%$ vs. $7 \%, p=0.01$; day $7: 46 \%$ vs. $22 \%, p<$ 0.001 ; day 14: $66 \%$ vs. $49 \%, p=0.001)$. Follow-up 
neurological outcomes remain pending for the STOP-BPD trial. Clarity regarding the efficacy and safety of late hydrocortisone will be bolstered by the ongoing "Hydrocortisone for BPD" trial, a large RCT by the NICHD's Neonatal Research Network on low-dose hydrocortisone $(18 \mathrm{mg} / \mathrm{kg}$ cumulative dose over 10 days) initiated at 14-28 days of age [45]. Outstanding questions regarding both the relative efficacy and neurologic impact of dexamethasone and hydrocortisone highlight the urgent need for direct comparison of relatively high-dose hydrocortisone to low-dose dexamethasone in randomized trials [46, 47].

A specific meta-analysis of trials for both early and late corticosteroids demonstrates a strong correlation between baseline risk of BPD in the study population and risk of the composite outcome of death or CP [48]. Randomization of infants with a low baseline risk of moderate-to-severe BPD $(<33 \%)$ produces increased death or $\mathrm{CP}$ in the treatment group. In infants with a high baseline risk $(>60 \%)$, the composite outcome of death or $\mathrm{CP}$ is reduced by corticosteroid treatment. In this context, many clinicians utilize corticosteroids (either low-dose dexamethasone or highdose hydrocortisone) in infants who require persistent invasive mechanical ventilation in the early evolving phase of BPD. One way to identify high-risk infants who can be considered for corticosteroid use is with the Neonatal BPD Outcome Estimator [49]. This tool provides an objective risk of BPD or death at various postnatal time points depending on the infant's GA, weight, sex, race, and respiratory support. However, direct application of the evidence-based moderate-to-severe BPD threshold of $60 \%$ utilizing the Estimator is problematic since BPD risk decreases as clinical status worsens due to increased mortality risk $[50,51]$. Therefore, centers must individualize a treatment threshold that stratifies outcomes (e.g., death vs. severe BPD) considering local risk profiles and family priorities.

\section{Late use of postnatal corticosteroids ( $>7$ days of age): late evolving BPD (28 days to 36 weeks' PMA)}

There are few data to guide the treatment of infants older than 1-month postnatal age but not yet 36 weeks' PMA. However, from the National Institutes of Health's Prematurity and Respiratory Outcomes Program, dexamethasone is primarily used between 6 and 7 weeks' postnatal age and a median of 32-33 weeks' PMA [52]. A single-center retrospective study $(N=98)$ was performed to assess short-term respiratory outcomes comparing 9-10 day courses of dexamethasone (initial dose $0.2 \mathrm{mg} / \mathrm{kg} / \mathrm{day}$ ), hydrocortisone (initial dose $4.8 \mathrm{mg} / \mathrm{kg} / \mathrm{day}$ ), and methylprednisolone (initial dose $2.4 \mathrm{mg} / \mathrm{kg} / \mathrm{day}$ ). The study included intubated preterm infants who received initial steroids at a mean postnatal age of 27 days with an average
30 weeks' PMA. The results of the study suggest that dexamethasone most effectively facilitates extubation by day 7 of treatment (59\% vs. $44 \%$ vs. $41 \%$, respectively; $p=$ 0.03 ); however, no difference was detected in the rates of BPD between groups [53]. Further studies in this time period could help guide clinicians on the timing and effectiveness of corticosteroids to facilitate extubation, reduce the severity of BPD, and assess improvements in morbidities (e.g., NDI, CP).

\section{Late use of postnatal corticosteroids ( $>7$ days of age): established BPD ( $\geq 36$ weeks' PMA)}

The desire to harness dexamethasone's potent antiinflammatory activity with some mineralocorticoid effect has led to the exploration of prednisolone and methylprednisolone (synthetic glucocorticoids with predominantly, but not exclusively, glucocorticoid activity). No prospective RCT has been performed to evaluate their effectiveness for the prevention or treatment of BPD. Retrospective studies have reported prednisolone's potential utility for infants already $>36$ weeks' PMA with varying degrees of established BPD. Bhandari et al. administered prednisolone to infants $>36$ weeks' PMA (mean $~ 38$ weeks' PMA) on supplemental oxygen but not on mechanical ventilation at the time of trial enrollment (Table 2) [54]. This study demonstrated that prednisolone therapy facilitated the ability to wean off supplemental oxygen in $63 \%$ of treated infants. A retrospective study by Linafelter et al. administered a longer course of prednisolone (nominally 28 days, but the paper included only those who received $\geq 30$ days, median 67 days [Table 2]) and included infants with severe BPD, half of whom were mechanically ventilated and half on continuous positive airway pressure [55]. The investigators noted a significant decrease in respiratory support after one week of prednisolone therapy and noted no further benefits from the prolongation of therapy and significant linear growth impairment by week 4 . The vast majority of infants survived to discharge (86\%), although over half $(57 \%)$ required a tracheostomy. Both the Bhandari and Linafelter studies indicated a potential benefit for improving the respiratory status of infants with established BPD. However, both studies concluded that the benefits of prednisolone therapies may not be seen with a prolonged course or multiple courses. Likewise, benefits must be carefully measured against adverse effects.

ICS, such as beclomethasone, budesonide, and fluticasone, are also commonly used to treat evolving or established BPD [52]. ICS use is inversely related to GA (and directly related to duration of mechanical ventilation exposure) [56]. Although the use of late ICS is common in both neonatal intensive care unit and post-discharge settings (used in $9-14 \%$ of infants born $<29$ weeks' GA), the 
evidence supporting their efficacy is minimal [57]. Yuskel et al. demonstrated the potential utility of beclomethasone in a small randomized trial of preterm infants $(N=18)$, documenting reductions in coughing and wheezing late in the first year of life, and an increase in functional residual capacity after 6 weeks of therapy [58]. However, the utility of ICS in hospitalized infants and/or for a prolonged duration is less clear. Dugas et al. compared fluticasone vs. placebo for a total of 4 weeks in 32 infants born $<32$ weeks' GA with established moderate BPD (requiring supplemental oxygen with a fraction of inspired oxygen $>0.25$ ) at postnatal ages between 28 and 60 days [59]. The study concluded that fluticasone does not reduce invasive mechanical ventilation duration or the need for supplemental oxygen use [59]. A prolonged 1-year course of fluticasone or placebo was also evaluated by Beresford et al. in 30 infants at 36 weeks' PMA with established BPD. This study reported no difference in respiratory symptoms, duration of supplemental oxygen use, respiratory illnesses, or need for hospitalization [60]. At this moment, although there is limited supporting evidence for the use of ICS, clinicians have few therapeutic options for managing ongoing pulmonary inflammation in preterm infants.

\section{Management of adrenal suppression}

In examining the highlighted trials above, we note that as postnatal age and PMA increase, the treatment regimens used both increased initial doses and cumulative doses (Fig. 3) of steroids. The cumulative steroid dose can be very high, particularly if infants are exposed to more than one regimen or stall in their glucocorticoid weaning due to

\section{Total Exposure of Preterm Infants to Corticosteroids}

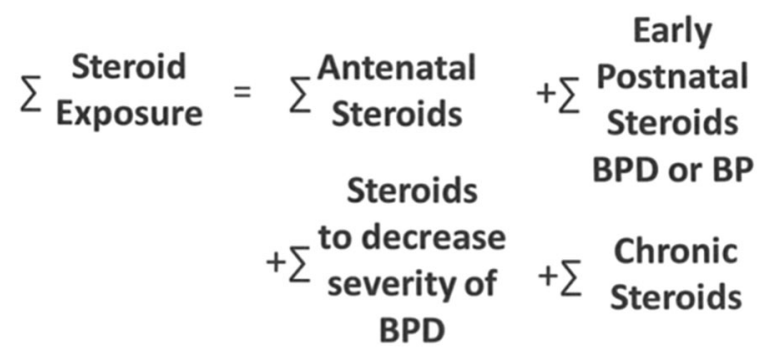

\section{Cumulative exposure is seldom considered when initiating a new steroid course}

Fig. 3 As a concept, any study trying to identify adverse associations of steroids needs to consider all steroids to which the infant has been exposed. Often antenatal steroid exposure is not considered. BPD bronchopulmonary dysplasia, BP blood pressure. failure to meet clinical outcomes, depending on the cumulative exposure and duration of the corticosteroid course(s) (Fig. 4). Infants are at risk for secondary adrenal insufficiency if the infant has been given supraphysiologic glucocorticoid doses for more than 14 days [61]. Exogenous supraphysiologic glucocorticoids cause secondary adrenal insufficiency through a myriad of effects on the HPA axis, the physiologic regulator of endogenous glucocorticoid production (Fig. 5). The hypothalamus releases corticotropin releasing hormone $(\mathrm{CRH})$ from neurons in the paraventricular nucleus. $\mathrm{CRH}$ then stimulates transcription of pro-opiomelanocortin from the anterior pituitary gland corticotropes. A series of proteolytic cleavages leads to the production of adrenocorticotropic hormone (ACTH). ACTH then stimulates the adrenal gland to produce cortisol. ACTH upregulates cortisol production acutely by increasing the available cholesterol substrate pool and stimulating the synthesis of the steroidogenic acute regulatory protein responsible for transporting cholesterol into the mitochondria for steroidogenesis to occur.

Prolonged exposure to glucocorticoids affects all levels of the HPA axis. Supraphysiologic steroid exposure blocks the hypothalamic neurons' ability to produce and store $\mathrm{CRH}$, and $\mathrm{CRH}$ receptor numbers are decreased in the pituitary gland. In addition, glucocorticoid therapy suppresses both pro-opiomelanocortin production and storage of ACTH. With long-term chronic suppression of ACTH release, there is a diminished transcription of enzymes involved in the biosynthesis of glucocorticoids. The adrenal reserve is lost, leading to the risk of an adrenal crisis after exogenous glucocorticoid treatment is discontinued. In addition, low ACTH levels can result in atrophy of the adrenal glands, as ACTH has a trophic effect on adrenocortical growth [62].

Recovery of the HPA axis after glucocorticoid exposure is dependent on multiple factors, including the total dose, potency, and length of glucocorticoid exposure. Experts generally agree that adrenal suppression is unlikely to occur in a patient treated with glucocorticoid for $<10$ days [61, 63]. However, if an infant develops unexplained hypoglycemia, electrolyte abnormalities, or refractory hypotension, stress dose treatment with hydrocortisone ( $\sim 50 \mathrm{mg} / \mathrm{m}^{2} /$ day) should be strongly considered [15]. For infants who have been glucocorticoid -exposed for $>14$ days, the risk of HPA axis suppression is high. Studies in VLBW premature infants treated with a 3-week course of dexamethasone demonstrated differential recovery of the axis, with the hypothalamic-pituitary signaling (measured by $\mathrm{CRH}$ testing) recovering earlier than adrenal gland function (measured by ACTH stimulation testing) [64, 65]. This indicates that infants are at risk for an adrenal crisis following glucocorticoid therapy and will require stress dose steroids during critical illness, with or without fever, 


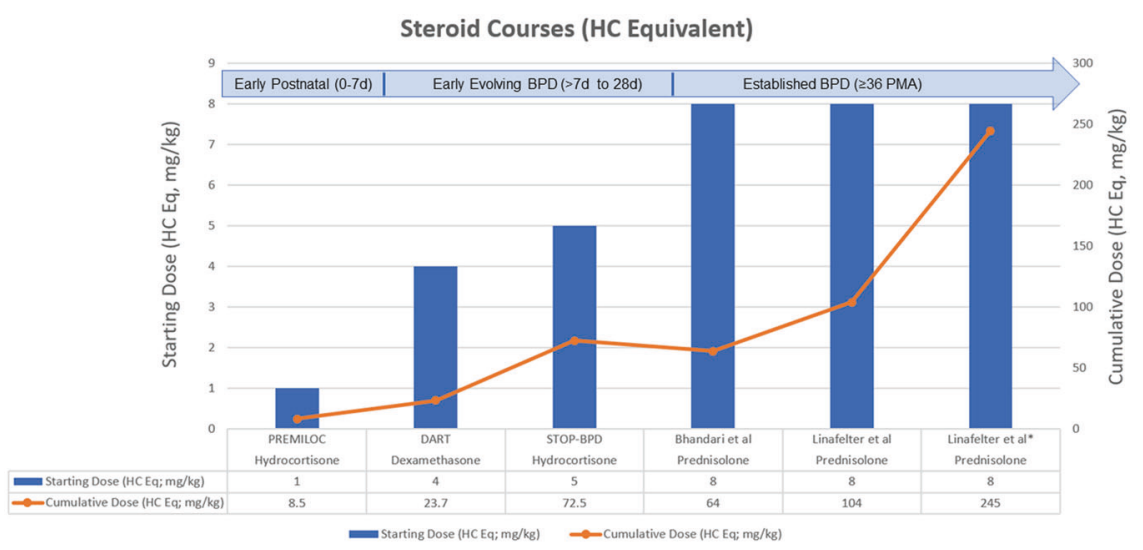

Fig. 4 Hydrocortisone equivalence for protocolized systemic postnatal corticosteroids described in respective studies. Linafelter has two different cumulative doses: $104 \mathrm{mg} / \mathrm{kg}$ represents the amount over 28 days as described by the protocol in the study; $* 245 \mathrm{mg} / \mathrm{kg}$ represents the actual reported median hydrocortisone equivalent used in the babies in that study (prednisolone cumulative dose of $61 \mathrm{mg} / \mathrm{kg}$ ) [55]. The blue bars/ starting dose uses the left vertical axis while the orange line/cumulative dose uses the right vertical axis.

Hypothalamus GR (MR)

Anterior Pituitary GR (MR)

\section{Adrenal Cortex}

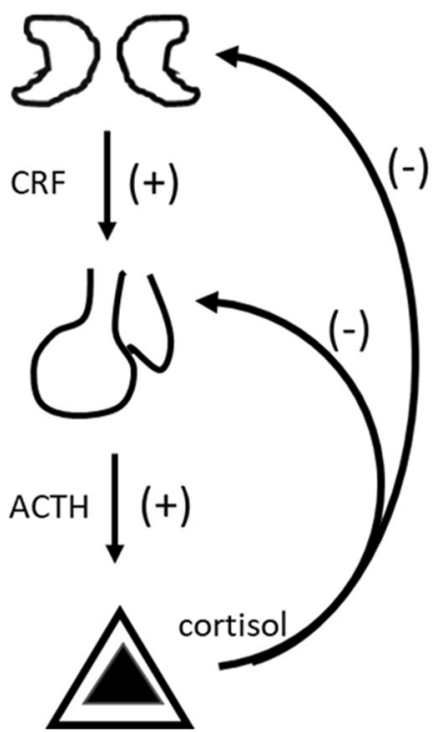

Fig. 5 Hypothalamic-pituitary-adrenal axis. Cortisol exerts negative feedback on both the hypothalamus and anterior pituitary, leading to decrease in both corticotropin releasing factor (CRF) and adrenocorticotropic hormone (ACTH) predominantly through action on the glucocorticoid receptor (GR). When cortisol levels drop or there is a physiologic need for cortisol release, CRF and ACTH levels rise, stimulating the secretion and production of cortisol by the adrenal gland. Mineralocorticoid receptors (MR) are expressed at low levels in the hypothalamus and anterior pituitary.

and for surgical procedures [61, 63]. Follow-up evaluation of the HPA axis by provocative testing (see below) will determine when stress dose steroids with an acute illness/ event can be discontinued.

\section{Proposed glucocorticoid weaning protocol}

For neonates who have been treated with supraphysiologic dose glucocorticoids for $<14$ days, tapering the glucocorticoid therapy for the final days of treatment may decrease the risk of adrenal suppression. This strategy is part of the regimens used in most postnatal corticosteroid studies (Table 2). While there are no specific guidelines for glucocorticoid weaning, we propose a protocol (Table 3). It should be noted that methylprednisolone, prednisolone, and dexamethasone are $4 x, 5 x$, and $25 x$ more potent than hydrocortisone, respectively (Table 1) [5]. These potencies are considered when developing glucocorticoid weaning plans, as the goal is to reach a "maintenance" glucocorticoid dose of $8-10 \mathrm{mg} / \mathrm{m}^{2} /$ day hydrocortisone equivalent (HC Eq) (Table 3).

For infants who have been on a steroid course for 10-14 days (including taper time), glucocorticoids can be discontinued once the steroid course is completed (Table 3). These infants generally do not require follow-up with pediatric endocrinologists. However, they should be monitored closely for signs and symptoms of adrenal insufficiency, including hypoglycemia, hypotension, and hyponatremia, as well as lethargy, poor feeding, and listlessness. Glucocorticoids are essential for free water excretion; thus, patients with central adrenal insufficiency may develop fluid retention and hyponatremia $[66,67]$. If these symptoms develop, the infant should be started on maintenance hydrocortisone $\left(8-10 \mathrm{mg} / \mathrm{m}^{2} /\right.$ day divided BID) and referred to pediatric endocrinology for further management. If the infant is discharged within 14 days of the steroid treatment, pediatrician evaluation post-discharge is prudent to ensure adequate feeding, growth, and developmental progress (Table 4) [68].

Infants who have been on steroids for $\geq 14$ days are at high risk for adrenal insufficiency [61, 63]. The suggested weaning protocol (Table 3) has been used for several years at Rainbow Babies \& Children's Hospital. A similar practice has been used for over a decade at the University at 
Table 3 Recommendations for weaning glucocorticoids (treatment $>14$ days).

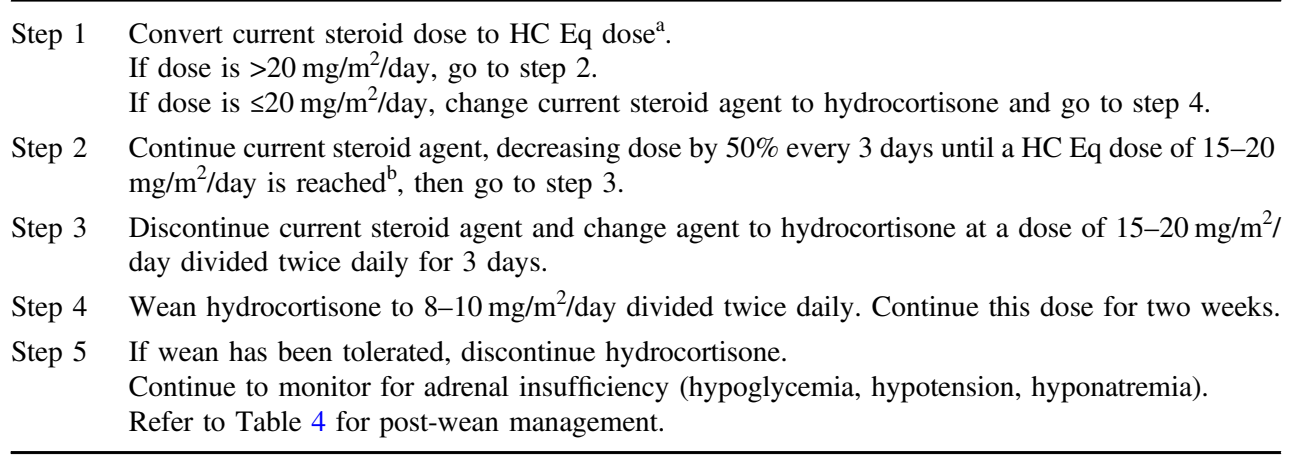
${ }^{\mathrm{a}} \mathrm{HC}$ Eq dose, hydrocortisone equivalency dose $\left(\mathrm{mg} / \mathrm{m}^{2} /\right.$ day $)$.

${ }^{\mathrm{b}}$ If the infant has any evidence of adrenal insufficiency during the wean, the dose can be increased back to the previously tolerated dose and the time between weans can be extended by an additional 3-6 days.
Buffalo. In our experience, the protocol is safe and adaptable for inpatient use. The initial step is to determine the current $\mathrm{HC}$ Eq dose that the baby is receiving at the start of the glucocorticoid wean (converting to $\mathrm{HC}$ dose in $\mathrm{mg} / \mathrm{m}^{2}$ / day). Using the original glucocorticoid formulation, decrease the dose by $50 \%$ every 3 days until the $\mathrm{HC} \mathrm{Eq}$ dose is $15-20 \mathrm{mg} / \mathrm{m}^{2} /$ day. At that point, convert over to hydrocortisone administered as twice-daily dosing for 3 days. Then decrease to $8-10 \mathrm{mg} / \mathrm{m}^{2} /$ day hydrocortisone and administer for 2 weeks ("maintenance" hydrocortisone) before discontinuation. During this time, especially with twice-daily dosing and lower exogenous HC levels, the HPA axis has an opportunity to initiate endogenous cortisol production. If the infant has any evidence of adrenal insufficiency during the weaning process, the dose can be increased to the previously tolerated dose, and the frequency of dosing weans can be extended by an additional 3-6 days.

Once the steroid wean is complete, the infant should be monitored closely for signs of adrenal insufficiency. The team also needs to determine if and when the infant should undergo provocative stimulation of the adrenal gland to evaluate the recovery of the HPA axis [61, 63]. Based on our collective experience and recommendations, Table 4 guides management with respect to the involvement of a pediatric endocrinologist and the need for a stimulation test. The length of steroid exposure and the anticipated observation period in the hospital after the steroid wean are considered when making this decision. The recommendations in Table 4 have been followed since 2008 and 2011, respectively, in the NICUs at Women and Children's Hospital of Buffalo and Medical University of South Carolina and have worked well.

To assess adrenal gland function following prolonged glucocorticoid exposure, an ACTH stimulation test is conducted by administering $1 \mathrm{mcg}$ dose of cosyntropin (i.e., synthetic ACTH) intravenously. Cortisol levels are measured at baseline and then 20 and $30 \mathrm{~min}$ after the administration of synthetic ACTH [69]. Some testing protocols measure cortisol at only 30 and $60 \mathrm{~min}$; however, cortisol generally peaks earlier in the low-dose ACTH test [70]. A peak of $>18 \mathrm{mcg} / \mathrm{dl}$ at any of the time points indicates recovery of the HPA axis. Although obtaining cortisol levels in infants after being weaned to maintenance (or off) hydrocortisone might decrease the number of ACTH stimulation tests, our collective recommendation is that obtaining a pre-8 a.m. cortisol level is not useful in this patient population. This is because infants, particularly those in an intensive care setting, have not established a diurnal rhythm for cortisol production [71, 72]. We recommend consultation with a pediatric endocrinologist for any patient with steroid exposure $>14$ days (Table 4). The pediatric endocrinology team can assist with the interpretation of adrenal function testing, can provide stress dose counseling to families as needed, and plan outpatient follow up as needed.

\section{Stress dose teaching}

Even with the recommended glucocorticoid weans, some neonates will have either confirmed or presumed ongoing secondary adrenal insufficiency at the time of discharge. For these patients, a discharge plan should be developed with pediatric endocrinology colleagues. Adrenal crisis is precipitated by physiologic stress, including fever, acute respiratory and gastrointestinal illnesses, trauma, and/or surgery. In such a situation, the family should be instructed in advance to administer a "stress dose" of hydrocortisone, teaching that should be provided by a pediatric endocrinologist. The daily oral emergency hydrocortisone dose is $50 \mathrm{mg} / \mathrm{m}^{2} /$ day, divided and administered three times per day. Hydrocortisone is commercially available as a tablet, which may be crushed and mixed with a small amount of formula. Oral suspensions are also commonly used and can be requested from a compounding pharmacy. Regardless of the formulation used, parents should be 


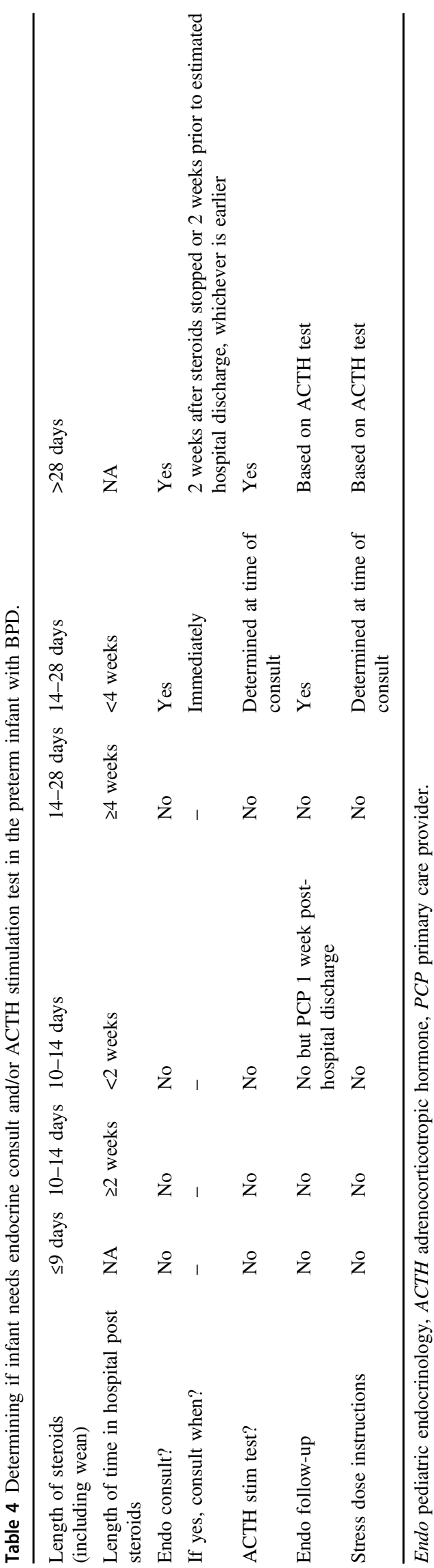

taught when and how much hydrocortisone should be administered, and instructed to monitor the expiration date of the formulation. Families can also be provided an "Emergency Stress Dose Letter" (provided as an example, Supplementary Document) that provides the following relevant information: the diagnosis, how to contact the endocrinologist, appropriate oral dosing, and intravenous doses of hydrocortisone that should be administered in an emergent situation such as acute illness, injury, or surgery. This letter also guides future medical professionals to provide appropriate care for an infant during the recovery phase of secondary adrenal insufficiency. Infants discharged from the NICU with an ongoing diagnosis of secondary adrenal insufficiency should be scheduled for outpatient follow-up with pediatric endocrinology. The endocrinology team will adjust hydrocortisone stress doses for growth, reinforce stress dosing issues, assess recovery of the HPA axis and answer guardian or caretaker questions.

The previous recommendations are based on a protocol developed collaboratively between neonatology and endocrinology in Buffalo in 2008, which avoids routine cortisol measurements. Anecdotally this protocol has worked well and been safe for over a decade.

\section{Summary}

The development of BPD is multidimensional. The imbalance of pro-inflammatory and anti-inflammatory pathways contributing to the lung injury can be attributed to different settings: prenatal (e.g., chorioamnionitis) and postnatal (e.g., mechanical ventilation, relative hyperoxia, sepsis). Systemic corticosteroids have anti-inflammatory properties and provide clinicians with options to prevent, treat, or mitigate the lung injury.

Dexamethasone and inhaled budesonide are not recommended for use before 7 days of age. Although early dexamethasone significantly decreases the combined outcome of death or BPD at 36 weeks' PMA, this benefit is outweighed by an increased associated risk of gastrointestinal perforation and NDI. Similarly, although inhaled budesonide decreases the risk of BPD, it has been shown to increase mortality. Favorable results from the PREMILOC trial suggest promise for early hydrocortisone use. Clinicians at centers with high BPD rates could consider early prophylactic hydrocortisone due to its benefit of improved survival without BPD at 36 weeks' PMA without any significant NDI. If early hydrocortisone is used, concurrent treatment with NSAIDs must be avoided to reduce the risk of gastrointestinal perforation.

Infants with either evolving or established BPD may benefit from late postnatal corticosteroids. Late use ( $>7$ days of age) of corticosteroids demonstrates the benefit of facilitating extubation or decreasing respiratory support without 
NDI. However, a consensus approach balancing the benefits and risks of corticosteroid treatment in early evolving BPD does not exist. There are also no prospective trials comparing dexamethasone to hydrocortisone directly. The relative benefits (timing of extubation, prevention of BPD, and late pulmonary complications) and risks (long-term NDI) of high-dose hydrocortisone compared to low-dose dexamethasone remain a research priority. Both hydrocortisone (utilizing the STOP-BPD regimen) and dexamethasone (utilizing the DART regimen) facilitate extubation without NDI. In our practice, if a steroid is to be given before day 21 , we consider hydrocortisone, rather than dexamethasone, due to dexamethasone's neurological harm observed in animal models and retrospective data before that time [46, 47, 73]. After 21 days of age, based on clear efficacy in this window of lung disease, dexamethasone can be used with less concern for neurologic harm.

There is currently a research gap for infants with later evolving BPD (28 days of age to 36 weeks' PMA). In this period, the evidence for BPD management with corticosteroids is minimal. For infants born at 24 weeks' GA, for example, this period of eight weeks (28 days of age to 36 weeks' PMA) may represent a critical stage for BPD evolution. In our practice, we continue to utilize the DART regimen in this period. However, any further relevant steroid treatment data available for infants $>28$ days of age will help clinicians, and prospective studies are highly desirable.

For infants with established severe BPD, prednisolone may be used, but data are based only on retrospective analyses. In our practice, we tend to utilize the shorter Bhandari et al. prednisolone dosing primarily in ventilated infants who may be able to extubate or who have a concurrent severe exacerbation of their underlying lung disease on high respiratory support. When prolonged prednisolone courses in the Linafelter et al. age range are needed (i.e., $>30$ days), we feel that collaboration with a pediatric pulmonologist is warranted. These longer courses of corticosteroids should be tailored to the infant's goals of therapy and response. Likewise, monitoring for adverse effects, including growth failure, should be assessed regularly as a multidisciplinary team.

Iatrogenic adrenal insufficiency is a side effect of this glucocorticoid treatment and must be managed appropriately. By recognizing the different potencies of glucocorticoids and using rational weaning protocols, recovery of the HPA axis is possible in the hospital. If this is not an option, families should be educated on recognizing and treating adrenal insufficiency in order to decrease the risk of morbidity. The neonatal care team plays a definitive role in the recognition and management of secondary adrenal insufficiency while providing reassurance to caregivers that this condition generally resolves as their child matures.
Since systemic steroids are used commonly in premature infants in various circumstances, including in the management of BPD, we need to be strategic in how we safely support the recovery of the HPA axis for these babies.

Acknowledgements We thank Pediatric Endocrinology and Neonatology from University of Buffalo and Rainbow Babies and Children's Hospital for sharing and input about the steroid weaning protocol.

Author contributions RMR provided the leadership, senior directorship, and responsibility of the review outline and concept. RKD, ZTH, AHJ, JM, LDM, CCM, RMR, and ES contributed to the drafting, reviewing, and revision of the manuscript. All authors approved the final manuscript as submitted and agree to be accountable for all aspects of the work.

\section{Compliance with ethical standards}

Conflict of interest The authors declare no competing interests.

Publisher's note Springer Nature remains neutral with regard to jurisdictional claims in published maps and institutional affiliations.

\section{References}

1. Jobe AH. Postnatal corticosteroids for bronchopulmonary dysplasia. Clin Perinatol. 2009;36:177-88.

2. Ryan RM, Ahmed Q, Lakshminrusimha S. Inflammatory mediators in the immunobiology of bronchopulmonary dysplasia. Clin Rev allergy Immunol. 2008;34:174-90.

3. Higgins RD, Saade G, Polin RA, Grobman WA, Buhimschi IA, Watterberg $\mathrm{K}$, et al. Evaluation and management of women and newborns with a maternal diagnosis of chorioamnionitis: summary of a workshop. Obstet Gynecol. 2016;127:426-36.

4. Williams DM. Clinical pharmacology of corticosteroids. Respiratory care. 2018;63:655-70.

5. Czock D, Keller F, Rasche FM, Häussler U. Pharmacokinetics and pharmacodynamics of systemically administered glucocorticoids. Clin Pharmacokinetics. 2005;44:61-98.

6. Raissy HH, Kelly HW, Harkins M, Szefler SJ. Inhaled corticosteroids in lung diseases. Am $\mathrm{J}$ respiratory Crit care Med. 2013;187:798-803.

7. Schimmer BP, Funder JW. ACTH, adrenal steroids, and the adrenal cortex. In: Brunton LL, Hilal DR, Knollmann BC, editors. Goodman \& Gilman's: the pharmacological basis of therapeutics. 13th ed. New York, NY: McGraw-Hill Education; 2017.

8. McKusick VA, Kniffin CA. Online Mendelian Inheritance in Man. Baltimore, MD: Johns Hopkins University. 2007. https:// omim.org/.

9. Brunton LLLJ, Parker KL. Goodman and Gilmans's the pharmacological basis of therapeutics. 11th ed. New York, NY: The McGraw-Hill Companies Inc; 2006.

10. Hanna CE, Jett PL, Laird MR, Mandel SH, LaFranchi SH, Reynolds JW. Corticosteroid binding globulin, total serum cortisol, and stress in extremely low-birth-weight infants. Am J Perinatol. 1997;14:201-4.

11. Scott SM, Wells L. Corticosteroid-binding globulin in preterm infants in an intensive care unit. Horm Res. 1995;44:218-21.

12. McKay LI, Cidlowski JA. Physiologic and pharmacologic effects of corticosteroids. In: Kufe DW, Pollock RE, Weichselbaum RR, et al., editors. Holland-Frei Cancer Medicine. 6th ed. Hamilton, ON: BC Decker; 2003. 
13. Fuller PJ, Young MJ. Mechanisms of mineralocorticoid action. Hypertension. 2005;46:1227-35.

14. Liu D, Ahmet A, Ward L, Krishnamoorthy P, Mandelcorn ED, Leigh $\mathrm{R}$, et al. A practical guide to the monitoring and management of the complications of systemic corticosteroid therapy. Allergy, Asthma, Clin Immunol. 2013;9:30.

15. Shulman DI, Palmert MR, Kemp SF. Adrenal insufficiency: still a cause of morbidity and death in childhood. Pediatrics. 2007;119: e484-94.

16. NCfB Information. PubChem Compound Summary for CID 5754, Hydrocortisone. 2004. https://pubchem.ncbi.nlm.nih.gov/compound/ Hydrocortisone.

17. Ahmet A, Kim H, Spier S. Adrenal suppression: a practical guide to the screening and management of this under-recognized complication of inhaled corticosteroid therapy. Allergy, Asthma, Clin Immunol. 2011;7:13.

18. Timmermans S, Souffriau J, Libert C. A general introduction to glucocorticoid biology. Front Immunol. 2019;10:1545.

19. André $\mathrm{P}$, Thébaud $\mathrm{B}$, Odièvre $\mathrm{MH}$, Razafimahefa $\mathrm{H}$, Zupan V, Dehan $M$, et al. Methylprednisolone, an alternative to dexamethasone in very premature infants at risk of chronic lung disease. Intensive Care Med. 2000;26:1496-500.

20. Littenberg B, Gluck EH. A controlled trial of methylprednisolone in the emergency treatment of acute asthma. N Engl J Med. 1986;314:150-2.

21. Meduri GU, Headley AS, Golden E, Carson SJ, Umberger RA, Kelso T, et al. Effect of prolonged methylprednisolone therapy in unresolving acute respiratory distress syndrome: a randomized controlled trial. JAMA. 1998;280:159-65.

22. Doyle LW, Ehrenkranz RA, Halliday HL. Early ( $<8$ days) postnatal corticosteroids for preventing chronic lung disease in preterm infants. Cochrane Database Syst Rev. 2014;5:Cd001146.

23. Doyle LW, Cheong JL, Ehrenkranz RA, Halliday HL. Late (>7 days) systemic postnatal corticosteroids for prevention of bronchopulmonary dysplasia in preterm infants. Cochrane Database Syst Rev. 2017;10:Cd001145.

24. Watterberg K. Fetal adrenal development: implications for lung development and postnatal disease. NeoReviews. 2006;7: e135-42.

25. Watterberg KLSS. Evidence of early adrenal insufficiency in babies who develop bronchopulmonary dysplasia. Pediatrics. 1995;95:120-5.

26. Shinwell ES, Karplus M, Reich D, Weintraub Z, Blazer S, Bader $\mathrm{D}$, et al. Early postnatal dexamethasone treatment and increased incidence of cerebral palsy. Arch Dis Child Fetal Neonatal Ed. 2000;83:F177-81.

27. Ng PC. The effectiveness and side effects of dexamethasone in preterm infants with bronchopulmonary dysplasia. Arch Dis Child. 1993;68:330-6.

28. Baud O, Maury L, Lebail F, Ramful D, El Moussawi F, Nicaise C, et al. Effect of early low-dose hydrocortisone on survival without bronchopulmonary dysplasia in extremely preterm infants (PREMILOC): a double-blind, placebo-controlled, multicentre, randomised trial. Lancet. 2016;387:1827-36.

29. Baud O, Trousson C, Biran V, Leroy E, Mohamed D, Alberti C. Association between early low-dose hydrocortisone therapy in extremely preterm neonates and neurodevelopmental outcomes at 2 years of age. JAMA. 2017;317:1329-37.

30. Baud O, Trousson C, Biran V, Leroy E, Mohamed D, Alberti C. Two-year neurodevelopmental outcomes of extremely preterm infants treated with early hydrocortisone: treatment effect according to gestational age at birth. Arch Dis Child Fetal Neonatal Ed. 2019;104:F30-f5.

31. Bassler D, Plavka R, Shinwell ES, Hallman M, Jarreau PH, Carnielli V, et al. Early inhaled budesonide for the prevention of bronchopulmonary dysplasia. N Engl J Med. 2015;373:1497-506.
32. Bassler D, Shinwell ES, Hallman M, Jarreau PH, Plavka R, Carnielli V, et al. Long-term effects of inhaled budesonide for bronchopulmonary dysplasia. N Engl J Med. 2018;378:148-57.

33. Delara M, Chauhan BF, Le ML, Abou-Setta AM, Zarychanski R, tJong GW. Efficacy and safety of pulmonary application of corticosteroids in preterm infants with respiratory distress syndrome: a systematic review and meta-analysis. Arch Dis Child Fetal Neonatal Ed. 2019;104:F137-f44.

34. Mazela J, Polin RA. Aerosol delivery to ventilated newborn infants: historical challenges and new directions. Eur J Pediatrics. 2011;170:433-44

35. Yeh TF, Lin HC, Chang CH, Wu TS, Su BH, Li TC, et al. Early intratracheal instillation of budesonide using surfactant as a vehicle to prevent chronic lung disease in preterm infants: a pilot study. Pediatrics. 2008;121:e1310-8.

36. Yeh TF, Chen CM, Wu SY, Husan Z, Li TC, Hsieh WS, et al. Intratracheal administration of budesonide/surfactant to prevent bronchopulmonary dysplasia. Am J Respiratory Crit Care Med. 2016;193:86-95.

37. NICHD. The Budesonide in Babies (BiB) Trial (BiB) Bethesda: Clinicaltrials.gov. 2020. https://clinicaltrials.gov/ct2/show/ NCT04545866.

38. O'Shea TM, Kothadia JM, Klinepeter KL, Goldstein DJ, Jackson BG, Weaver RG 3rd, et al. Randomized placebo-controlled trial of a 42-day tapering course of dexamethasone to reduce the duration of ventilator dependency in very low birth weight infants: outcome of study participants at 1-year adjusted age. Pediatrics. 1999;104:15-21.

39. O'Shea TM, Washburn LK, Nixon PA, Goldstein DJ. Follow-up of a randomized, placebo-controlled trial of dexamethasone to decrease the duration of ventilator dependency in very low birth weight infants: neurodevelopmental outcomes at 4 to 11 years of age. Pediatrics. 2007;120:594-602.

40. Onland W, De Jaegere AP, Offringa M, van Kaam AH. Effects of higher versus lower dexamethasone doses on pulmonary and neurodevelopmental sequelae in preterm infants at risk for chronic lung disease: a meta-analysis. Pediatrics. 2008;122:92-101.

41. Doyle LW, Davis PG, Morley CJ, McPhee A, Carlin JB. Lowdose dexamethasone facilitates extubation among chronically ventilator-dependent infants: a multicenter, international, randomized, controlled trial. Pediatrics. 2006;117:75-83.

42. Rademaker KJ, Groenendaal F, van Bel F, de Vries LS, Uiterwaal CSPM. The DART study of low-dose dexamethasone therapy. Pediatrics. 2007;120:689-90.

43. Doyle LW, Davis PG, Morley CJ, McPhee A, Carlin JB. Outcome at 2 years of age of infants from the DART study: a multicenter, international, randomized, controlled trial of low-dose dexamethasone. Pediatrics. 2007;119:716-21.

44. Onland W, Cools F, Kroon A, Rademaker K, Merkus MP, Dijk $\mathrm{PH}$, et al. Effect of hydrocortisone therapy initiated 7 to 14 days after birth on mortality or bronchopulmonary dysplasia among very preterm infants receiving mechanical ventilation: a randomized clinical trial. JAMA. 2019;321:354-63.

45. NIH. Hydrocortisone for BPD. Bethesda, MD: ClinicalTrials.gov; 2011. https://clinicaltrials.gov/ct2/show/NCT01353313.

46. van der Heide-Jalving M, Kamphuis PJ, van der Laan MJ, Bakker JM, Wiegant VM, Heijnen CJ, et al. Short- and long-term effects of neonatal glucocorticoid therapy: is hydrocortisone an alternative to dexamethasone? Acta Paediatr. 2003;92:827-35.

47. Hitzert MM, Benders MJ, Roescher AM, van Bel F, de Vries LS, Bos AF. Hydrocortisone vs. dexamethasone treatment for bronchopulmonary dysplasia and their effects on general movements in preterm infants. Pediatr Res. 2012;71:100-6.

48. Doyle LW, Halliday HL, Ehrenkranz RA, Davis PG, Sinclair JC. An update on the impact of postnatal systemic corticosteroids on mortality and cerebral palsy in preterm infants: effect modification 
by risk of bronchopulmonary dysplasia. J Pediatrics. 2014;165:1258-60.

49. NICHD. Neonatal BPD outcome estimator: infatns with GA 23-30 weeks \& birth weight 501-1249 g. Bethesda, MD: Neonatal Research Network; 2020. https://neonatal.rti.org/index.cfm.

50. Vesoulis ZA, McPherson CC, Whitehead HV. Racial disparities in calculated risk for bronchopulmonary dysplasia: a dataset. Data Brief. 2020;30:105674.

51. Boland RA, Davis PG, Dawson JA, Doyle LW. Outcomes of infants born at 22-27 weeks' gestation in Victoria according to outborn/inborn birth status. Arch Dis Child Fetal Neonatal Ed. 2017;102:F153-f61.

52. Greenberg JM, Poindexter BB, Shaw PA, Bellamy SL, Keller RL, Moore PE, et al. Respiratory medication use in extremely premature ( $<29$ weeks) infants during initial NICU hospitalization: results from the prematurity and respiratory outcomes program. Pediatr Pulmonol. 2020;55:360-8.

53. Nath S, Reynolds AM, Lakshminrusimha S, Ma C, Hudak ML, Ryan RM. Retrospective analysis of short-term respiratory outcomes of three different steroids used in clinical practice in intubated preterm infants. Am J Perinatol. 2020;37:1425-31.

54. Bhandari A, Schramm CM, Kimble C, Pappagallo M, Hussain N. Effect of a short course of prednisolone in infants with oxygendependent bronchopulmonary dysplasia. Pediatrics. 2008;121: e344-9.

55. Linafelter A, Cuna A, Liu C, Quigley A, Truog WE, Sampath V, et al. Extended course of prednisolone in infants with severe bronchopulmonary dysplasia. Early Hum Dev. 2019;136:1-6.

56. Slaughter JL, Stenger MR, Reagan PB, Jadcherla SR. Utilization of inhaled corticosteroids for infants with bronchopulmonary dysplasia. PLoS ONE. 2014;9:e106838.

57. Ryan RM, Keller RL, Poindexter BB, D'Angio CT, Shaw PA, Bellamy SL, et al. Respiratory medications in infants $<29$ weeks during the first year postdischarge: the prematurity and respiratory outcomes program (PROP) consortium. J Pediatrics. 2019;208:e3.

58. Yuksel B, Greenough A. Randomised trial of inhaled steroids in preterm infants with respiratory symptoms at follow up. Thorax. 1992;47:910-3.

59. Dugas MA, Nguyen D, Frenette L, Lachance C, St-Onge O, Fougères $\mathrm{A}$, et al. Fluticasone inhalation in moderate cases of bronchopulmonary dysplasia. Pediatrics. 2005;115:e566-72.

60. Beresford MW, Primhak R, Subhedar NV, Shaw NJ. Randomised double blind placebo controlled trial of inhaled fluticasone propionate in infants with chronic lung disease. Arch Dis Child Fetal Neonatal Ed. 2002;87:F62-3.

61. Dempsher D. Adrenal and pituitary insufficiency in the neonate. NeoReviews. 2008;9:e72-7.
62. Younes AK, Younes NK. Recovery of steroid induced adrenal insufficiency. Transl Pediatrics. 2017;6:269-73.

63. Yiğit Ş, Türkmen M, Tuncer O, Taşkın E, Güran T, Abacı A, et al. Neonatal adrenal insufficiency: Turkish neonatal and pediatric endocrinology and diabetes societies consensus report. Turk Pediatr Ars. 2018;53:S239-43.

64. Ng PC, Wong GW, Lam CW, Lee CH, Fok TF, Wong MY, et al. Pituitary-adrenal suppression and recovery in preterm very low birth weight infants after dexamethasone treatment for bronchopulmonary dysplasia. J Clin Endocrinol Metab. 1997;82:2429-32.

65. Ng PC, Lam CW, Lee CH, Chan IH, Wong SP, Fok TF. Suppression and recovery of the hypothalamic function after highdose corticosteroid treatment in preterm infants. Neonatology. 2008;94:170-5.

66. Kleeman CR, Czaczkes JW, Cutler R. Mechanisms of impaired water excretion in adrenal and pituitary insufficiency. IV. Antidiuretic hormone in primary and secondary adrenal insufficiency. J Clin Investig. 1964;43:1641-8.

67. Chin HX, Quek TP, Leow MK. Central diabetes insipidus unmasked by corticosteroid therapy for cerebral metastases: beware the case with pituitary involvement and hypopituitarism. J R Coll Physicians Edinb. 2017;47:247-9.

68. Grossman AB. The diagnosis and management of central hypoadrenalism. J Clin Endocrinol Metab. 2010;95:4855-63.

69. Abdu TA, Elhadd TA, Neary R, Clayton RN. Comparison of the low dose short synacthen test ( 1 microg), the conventional dose short synacthen test (250 microg), and the insulin tolerance test for assessment of the hypothalamo-pituitary-adrenal axis in patients with pituitary disease. J Clin Endocrinol Metab. 1999;84:838-43.

70. Mongiò̀ LM, Condorelli RA, Barbagallo F, Cannarella R, La Vignera S, Calogero AE. Accuracy of the low-dose ACTH stimulation test for adrenal insufficiency diagnosis: a re-assessment of the cut-off value. J Clin Med. 2019;8:6.

71. Iwata O, Okamura H, Saitsu H, Saikusa M, Kanda H, Eshima N, et al. Diurnal cortisol changes in newborn infants suggesting entrainment of peripheral circadian clock in utero and at birth. J Clin Endocrinol Metab. 2013;98:E25-32.

72. Scott SM, Watterberg KL. Effect of gestational age, postnatal age, and illness on plasma cortisol concentrations in premature infants. Pediatr Res. 1995;37:112-6.

73. Huang CC, Lin HR, Liang YC, Hsu KS. Effects of neonatal corticosteroid treatment on hippocampal synaptic function. Pediatr Res. 2007;62:267-70.

74. Harmon HM, Jensen EA, Tan S, Chaudhary AS, Slaughter JL, Bell EF, et al. Timing of postnatal steroids for bronchopulmonary dysplasia: association with pulmonary and neurodevelopmental outcomes. J Perinatol. 2020;40:616-27. 